\title{
Increasing incidence of hypotension in the emergency department; a 12 year population-based cohort study
}

\author{
Jon G. Holler ${ }^{1 *}$, Daniel P. Henriksen ${ }^{2}$, Søren Mikkelsen ${ }^{3}$, Court Pedersen ${ }^{4}$ and Annmarie T. Lassen ${ }^{1}$
}

\begin{abstract}
Background: The epidemiology of hypotension as presenting symptom among patients in the Emergency Department (ED) is not clarified. The aim of this study was to describe the incidence, etiology, and overall mortality of hypotensive patients in the ED.

Methods: Population-based cohort study at an University Hospital ED in Denmark from January 1, 2000, to December 31, 2011. Patients aged $\geq 18$ years living in the hospital catchment area with a first time presentation to the ED with hypotension (systolic blood pressure (SBP) $\leq 100 \mathrm{~mm} \mathrm{Hg}$ ) were included.

Outcomes were annual incidence rates (IRs) per 100,000 person years at risk (pyar) and etiological characteristics by means of the International Classification of Diseases, Tenth Revision (ICD-10), as well as 7-day, 30-day, and 90-day all-cause mortality.

Results: We identified 3,268 of 438,198 (1 \%) cases with a mean overall IR of 125/100,000 pyar (95 \% Cl: 121-130). The IR increased $28 \%$ during the period (from 113 to 152 cases per 100,000 pyar). Patients $\geq 65$ years had the highest IR compared to age <65 years (rate ratio for men 6.3 (95 \% Cl: 5.6-7.1) and for women 4.2 ( $95 \%$ Cl: 3.6-4.9)). The etiology was highly diversified with trauma (17\%) and cardiovascular diseases (15\%) as the most common. The overall 7-day, 30-day and 90-day mortality rates were $15 \%$ (95\% Cl: 14-16), $22 \%$ (95\% Cl: 21-24) and $28 \%$ (95\% Cl: 27-30) respectively.
\end{abstract}

Conclusion: During 2000-2011 the overall incidence of ED hypotension increased and remained highest among the elderly with a diversified etiology and a 90-day all-cause mortality of $28 \%$.

Keywords: Hypotension, Epidemiology, Incidence, Etiology, Mortality, Emergency department

\section{Background}

Systolic blood pressure (SBP) is widely used in the initial triage of acutely ill patients and forms a basic part of the initial assessment of the circulation [1]. The presence of hypotension often signifies critical illness and several large multicenter studies have used the presence of hypotension as an inclusion criteria together with other variables [2]. These studies often focus on highly selected hypotensive patient populations in specialized treatment units, and the evidence gained is a reflection of this selection.

\footnotetext{
* Correspondence: Jon.Gitz.Holler@dadlnet.dk

${ }^{1}$ Department of Emergency Medicine, Odense University Hospital, Odense, Denmark

Full list of author information is available at the end of the article
}

During the past decade, research investigating annual trends in incidence rates (IR) of potentially hypotensive patients suggest opposite trends depending on the etiology and population of interest. While the annual IRs of sepsis seems to increase [3], the trend for myocardial infarction (MI) have decreased [4]. Whether population-based IRs and annual trends of primary undifferentiated hypotensive ED patients demonstrate dynamic trends, are not known. Previous estimates of hypotension in EDs rely mainly on hospital data samples that are weighted to extrapolate to national level estimates and are therefore vulnerable to sampling bias [5]. In general studies on this topic are limited, either by place of settings or selective inclusion criteria and conditions studied [6]. Population-based IRs of hypotension among ED patients is important to quantify 
as the presence of hypotension - even transient - is associated with worse outcomes and can therefore not be neglected [7]. The epidemiological knowledge gained, can serve as a foundation for future interventional studies in this critical population.

While ED visits in Denmark have been stable through recent years, ED visits among the ageing population are increasing [8]. Furthermore, time-sensitive critical illnesses (i.e. cardiogenic shock, severe sepsis, and the 'golden hour' of trauma), have increased the demand of prompt critical care recognition and delivery in the ED setting. Collectively, this adds to the hypothesis of a possible increasing trend in ED hypotension. We, therefore, conducted an ED populations-based cohort study to examine annual IRs in first time presentation of hypotension over a 12-year period from 2000-2011 and subsequent the etiology and short-term mortality.

\section{Methods}

\section{Study design and setting}

We conducted a population-based cohort study with data from the ED of Odense University Hospital, Denmark, during the period of 1th January 2000 to 31th December 2011 (12 years). Odense University Hospital is a 1,000-bed university teaching hospital representing all specialties including surgical, neurological, and general internal medical patients. The population served by this ED consists of four well-defined municipalities with a mixed rural-urban population of 290,000 persons. It is the only serving ED in this part of Denmark and provides primary 24-h acute medical care, with 48.000 annual visits.

\section{Selection of participants}

Adults (age $\geq 18$ years) were considered eligible when presenting to the ED with a SBP $\leq 100 \mathrm{~mm} \mathrm{Hg}$ registered within $3 \mathrm{~h}$ upon arrival. Based on a recent published study, examining SPB thresholds and mortality in our ED, we defined a SBP $\leq 100 \mathrm{~mm} \mathrm{Hg}$ as hypotension [9]. We used the Shock Index (SI) as a proxy for acute illness. SI is calculated as the ratio of heart rate to SBP and included as a categorical variable $(<0.7,0.7-1, \geq 1)[10]$. If a patient had multiple encounters with hypotension over the study period, only the first was included within the cohort. The primary date of contact defined the index date. Patients $<18$ years, patients residing outside the hospitals catchment area at the time of contact, and patients without a Danish personal identification number were excluded. We also excluded patients with a previous presentation of SBP $\leq 100 \mathrm{~mm} \mathrm{Hg}$. To minimize left sided censoring, patients who had visited the ED between 1 of January 1998 and 1 of January 2000 with hypotension were excluded as well. The background population, from which the cases were retrieved, was the composed general adult ( $\geq 18$ years) Danish citizens living in the hospitals catchment area.

\section{Variables and outcome measures}

The primary outcome was the IRs of hypotension (SBP $\leq$ $100 \mathrm{~mm} \mathrm{Hg}$ ) from 1st January 2000 to 31th January 2011 in the ED, both overall and by year. Secondary outcomes were etiological characteristics by means of major ICD-10 codes and the proportion of 7-day, 30-day, and 90-day allcause mortality. The primary exposure variable was the first recorded SBP value at presentation.

SBP was measured with an automated oscillometric device or manual cuff and sphygmomanometer. HR was measured with ECG, palpation or pulse oximetry. We also included information on the additional covariates; age, gender and time of contact during the day (07:00-14:59, 15:00-22:59, 23:00-06:59). Charlson comorbidity index (CCI; $0,1-2,>2$ ) was used as a marker of comorbid illness.

We defined etiology based on the primary ED diagnoses and the immediate ensuing hospital discharge diagnosis. These were assigned by physicians in the ED at discharge/referral to other departments and based on the International Classification of Diseases, Tenth Revision (ICD-10) (see below).

\section{Data sources and processing}

In Denmark every Danish citizen is assigned a unique 10digit civil personal registry number (PRN-number). This unique PRN-number enables accurate linkage between the Danish national registers [11]. True population-based studies are hereby possible as all patient contacts are registered and linked between all Danish registries using the patients unique PRN-number.

\section{The Danish national patient registry}

Since 1995 the Danish National Patient Registry has been covering all in and out patient clinic contacts at hospitals in Denmark assembling data regarding dates of admission, discharge, admitting departments and all primary and secondary discharge diagnoses (ICD-10 code system) from hospitals (except psychiatric departments and hospitals) [11]. By discharge every unique patient journey is assigned one primary diagnosis and one or more secondary diagnosis (up to 20 diagnoses) classified according to the ICD-10 system. We used discharge diagnoses from the previous 10 years in order to generate a CCI for each enrolled patient upon the index contact date as a proxy for comorbid illness.

\section{Database}

Since 1996 all patients records from the ED are registered electronically and available as patients record notes from the contact. As a part of the routine procedure, all patients presenting to the ED, except those with minor orthopedic complaints, had their vital signs measured and registered by a nurse at arrival. The record notes are 
available in text-format, in which vital parameters are consistently stated, including time of admission and time of measured SBP and HR. By electronic screening it was possible to identify and retrieve information on all patients with a measured and registered SBP $\leq 100 \mathrm{~mm} \mathrm{Hg}$ as well as the unique value of SPB and HR. The present data extraction process has been manually validated in 500 files with a sensitivity of $96 \%$ (95\% CI [91-99]) and a specificity of $100 \%$ (95\% CI [99-100]) for exact SBP, in the study by Kristensen et al. [9].

Data on municipality of residence, migration-, maritaland vital status, and date of birth were retrieved from The Danish Civil Registration System and linked to other registries and databases using the unique PNR-number [11].

\section{Other registers and databases}

We retrieved information regarding the annual mid-year population of persons 18 years old or older living in the hospitals catchment area (accessed September 2014 at Statistics Denmark website; http://www.statistikbanken.dk).

\section{Analysis}

Baseline characteristics were presented as medians and interquartile range (IQR) for continuous variables and categorical variables as numbers and percentages. We used the Chi-square test for categorical variables and the Kruskal-Wallis equality-of-populations rank test for continuous variables. Patients were followed from index date until the date of death, completion of 90 days follow-up, emigration, or December 31, 2011, whichever came first.

The crude annual IRs were calculated as the number of IRs per 100,000 pyar (age $\geq 18$ years) with the corresponding $95 \%$ confidence intervals $(95 \% \mathrm{CI}$ ) assuming a Poisson distribution. The annual IRs were adjusted using direct standardization to the sex- and age distribution of the municipalities of the EDs catchment area midyear population in the year 2000. The population was defined as contributing to one person-year at risk per resident per year in the analyses. The incidence rates were estimated and analyzed using a Poisson regression model. Age group, gender, calendar time in years, and interaction between age group and gender were used in the adjusted model. Calender time was entered in the model as a continuous variable. Age was divided into two predefined age intervals: 18-64 years and $\geq 65$ years. The Poisson model was assessed using the Hosmere Lemeshow goodness-of-fit test.

Etiological characteristics were categorized into major ICD-10 groups and calculated as frequencies and proportions based on primary registered conditions at discharge among all hypotensive patients as well as stratified into SBP intervals.

We constructed Kaplan-Meier curves and reported the all-cause 90-day mortality, stratified by SBP intervals. Comparisons between groups were evaluated with a log- rank test. Cuzick's test was used for trends in mortality between SBP intervals. All tests of significance were two-tailed, and $p$ values $<.05$ were considered significant. Missing values (ICD-codes; $n=2$ and HR; $n=128$ ) were excluded in the analysis of the specific variable. Statistical analyses were performed using Stata version 13.1 (Stata Corporation LP ${ }^{\oplus}$, Texas, USA).

\section{Ethics committee approval}

The study was approved by the Danish Data Protection Agency (J.nr 2008-58-0035) and the Danish Health and Medicines Authority (j.nr. 3-3013-205/1). In accordance with Danish law, observational studies performed in Denmark do not need approval from the Medical Ethics Committee. The study was conducted according to the STROBE statement.

\section{Results}

\section{Participants}

Among all patients the proportion of hypotensive patients was $1 \%(4,555 / 438,198)$ (Fig. 1). After exclusions (see Fig. 1 for details) we included 3,268 patients with a presentation of hypotension for further analysis. Of these the median age of 68 (IQR, 50-80), 1,602 (49\%) were males and 858 (26\%) presented with a Charlson Comorbidity Index greater than 2 (Table 1). Median SBP was 91 (IQR, 84-96). A SBP between 90 and $100 \mathrm{~mm} \mathrm{Hg}$ was present in 1,725 (53\%), 920 (28\%) had a SBP between 80 and $90 \mathrm{~mm} \mathrm{Hg}$ and 623 (19\%) had a SBP below $80 \mathrm{~mm} \mathrm{Hg}$ (Table 1). Most patients had their SBP measured within 30 min (92\%) after arrival and $50 \%$ were measured within 5 min. Contacts showed a diurnal rhythm with most patients arriving during the day and evening (see Fig. 2a and b).

\section{Incidence rate}

The annual crude IRs together with the standardized IRs of hypotension (SBP $\leq 100 \mathrm{~mm} \mathrm{Hg}$ ) and IRs of different levels of hypotension during the period 2000-2011 are shown in Fig. 3. The mean overall IR of hypotension during 20002011 was 125/100,000 pyar (95 \% CI: 121-130). The corresponding standardized mean IRs estimates was 122/ 100,000 person-years at risk (95\% CI: 118-127). The overall IR increased by 28 \% (95\% CI: 14-46 \%) from 113 in 2000 to 152 in 2011, with an average adjusted annual increase of $2 \%$ per year, (95\% CI: 1-3). Concordantly, the average annual increase using standardized estimates was 2 \% (95 \% CI: 1-3). The estimated IRs stratified by sex and age group with incidence rate ratios and crude IRs are shown in Fig. 4. Men aged $\geq 65$ had a six-time higher IR than men aged $<65$ years. We observed increasing IRs during the years 2008-2011, preceded by more or less stable rates during 2000-2008. When stratified on SBP intervals the overall mean IR of $90>\mathrm{SBP} \leq 100 \mathrm{~mm} \mathrm{Hg}$ was $66 /$ 100.000 pyar (95 \% CI: 63-69) and 35/100,000 pyar (95\% 


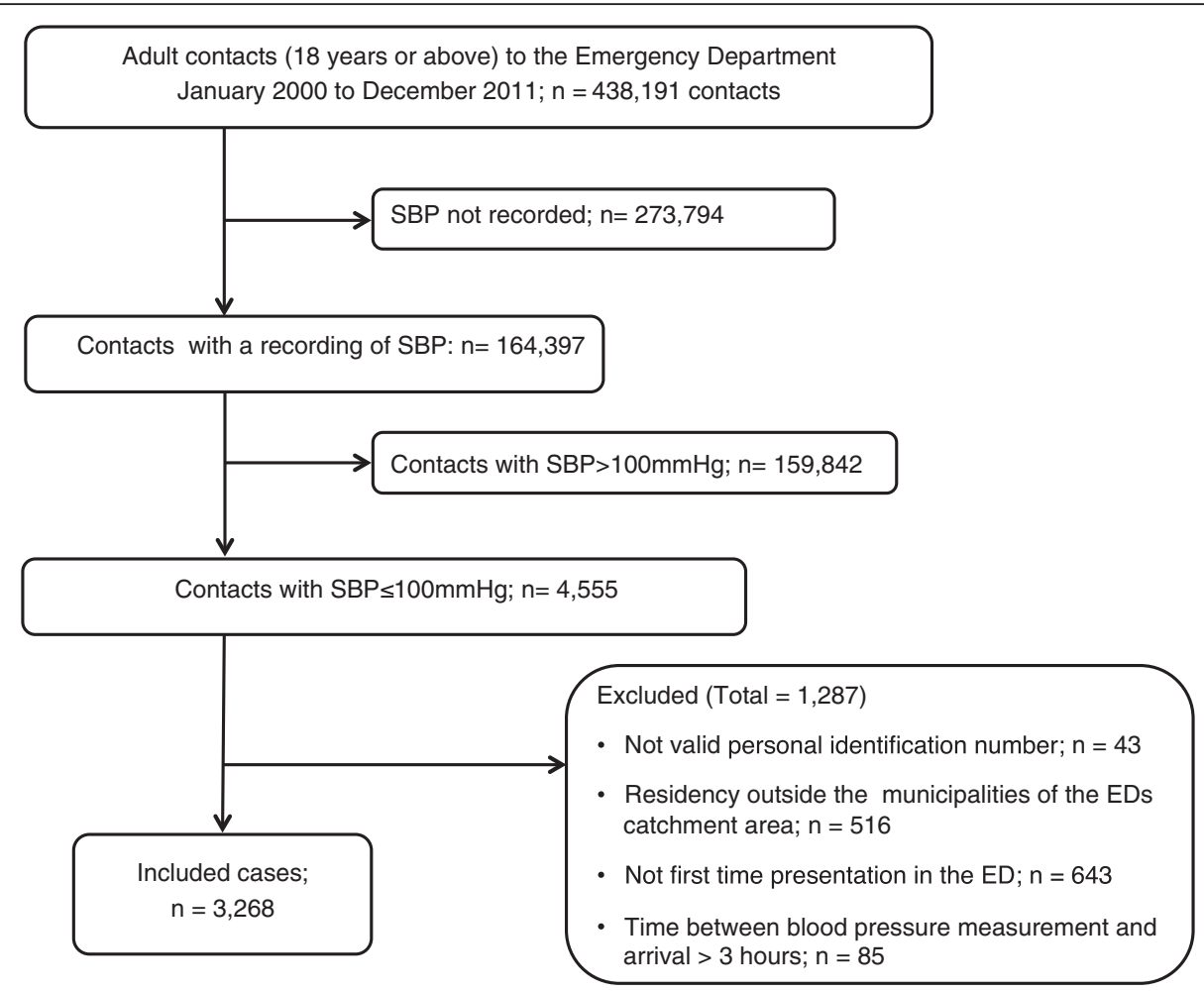

Fig. 1 Flow chart of patients recruited to the study

CI: 33-37) in the interval $80>\mathrm{SBP} \leq 90 \mathrm{~mm} \mathrm{Hg}$. SBP $\leq 80$ mm Hg was 24/100,000 pyar (95 \% CI: 22-26). Men aged $80+$ had the highest incidence rate with $797 / 100,000$ person-years at risk (95 \% CI: 718-884). See Fig. 5.

\section{Etiology}

The frequency and proportion of index and discharge conditions according to major $I C D$-10 discharge diagnoses are presented in Table 2. The major overall discharge condition were coded as "Injury, poisoning, and certain other consequences of external causes" (SOO-T98) (23\%) which were stratified into medical conditions (T36-T69, T80-T98) (8 \%) and trauma (SO0-T35, T70-T79, T90-T99) (17 \%)." Diseases of the circulatory system" (I00-199) (15\%), and "Symptoms, signs, and abnormal clinical and laboratory findings, not elsewhere classified" (ROO-R99) (14 \%). Analysis of discharge conditions across the period of observation revealed an increasing trend for infectious diseases (ICD10: A00-B99, $p=0.003$ ), respiratory diseases (ICD10: J00J99: $p=0.007$ ), genitourinary system (ICD10: N00-N99, $p=0.014)$, as well as symptoms, signs, and abnormal clinical and laboratory findings, not elsewhere classified (ICD10: R00-R99, $p=0.013$ ) see Table 3.

\section{Mortality}

The mean 7-day, 30-day and 90-day all-cause mortality was $15 \%$ (95 \% CI: 14-16), 22 \% (95\% CI: 21-24) and
$28 \%$ (95 \% CI: 27-30) respectively (Table 1). We observed an increase $(p<0.001)$ in mortality among patients SBP $\leq 80 \mathrm{~mm} \mathrm{Hg}$ (90-day mortality: $44 \%$ (95\% CI: 40-48)) compared to patients with SBP between $90>$ SBP $\leq 100 \mathrm{~mm} \mathrm{Hg}$ (90-day mortality: $21 \%$ (95\% CI: 19-23)). Kaplan-Meier curves are shown in Fig. 6 with the overall estimated probability of 90-day survival stratified into SBP intervals.

\section{Discussion}

This study provides population-based epidemiological characteristics of adult hypotensive patients arriving to a University ED in Denmark. Our results showed that a first time presentation of hypotension was a common finding in the ED with an increasing annual trend in IRs throughout the period 2000-2011. By means of discharge diagnoses the etiology was clearly diversified and the 90-day all-cause mortality was $28 \%$.

Our primary aim was to address the IR and trend of hypotension in the ED. In our study we have reported an overall mean IR of SBP $\leq 100 \mathrm{~mm} \mathrm{Hg}$ of 125/100,000 pyar. Comparing our IRs with other conditions suggest hypotension to be as common as first time hospitalization with myocardial infarction (MI) [12] and more common than ST-segment elevation MI [13]. While the IRs of MI have decreased during the past decades $[12,13]$ registered sepsis is on the rise [3]. The IRs of hypotension, in our 
Table 1 Baseline characteristics at time of arrival to the ED

\begin{tabular}{|c|c|c|c|c|}
\hline Variable & Total (\%) SBP $\leq 100 \mathrm{~mm} \mathrm{Hg}$ & $90>\mathrm{SBP} \leq 100 \mathrm{~mm} \mathrm{Hg}$ & $80>\mathrm{SBP} \leq 90 \mathrm{~mm} \mathrm{Hg}$ & $\mathrm{SBP} \leq 80 \mathrm{~mm} \mathrm{Hg}$ \\
\hline N (\%) & 3,268 (100 \%) & $1,725(100 \%)$ & $920(100 \%)$ & $623(100 \%)$ \\
\hline Age in years, Median (IQR) & $68(50-80)$ & $65(45-79)$ & $69(54-80)$ & $72(58-82)$ \\
\hline \multicolumn{5}{|l|}{ Sex (\%) } \\
\hline Male & $1,602(49 \%)$ & 792 (46 \%) & $462(50 \%)$ & 348 (56 \%) \\
\hline Female & $1,666(51 \%)$ & $933(54 \%)$ & $458(50 \%)$ & 275 (44 \%) \\
\hline \multicolumn{5}{|l|}{ Age in age groups, yr (\%) } \\
\hline $18-39$ & $509(16 \%)$ & $348(20 \%)$ & 117 (13\%) & $44(7 \%)$ \\
\hline $40-64$ & 942 (29\%) & $500(29 \%)$ & 275 (30 \%) & 167 (27 \%) \\
\hline $65-84$ & $1,313(40 \%)$ & $630(37 \%)$ & 388 (42 \%) & 295 (47 \%) \\
\hline $85+$ & $504(15 \%)$ & 247 (14 \%) & 140 (15\%) & 117 (19 \%) \\
\hline \multicolumn{5}{|l|}{ Charlson Comorbidity Index (\%) } \\
\hline 0 & $1,291(40 \%)$ & 739 (43 \%) & 336 (37 \%) & 216 (35 \%) \\
\hline 1 to 2 & $1,119(34 \%)$ & $572(33 \%)$ & 334 (36 \%) & $213(34 \%)$ \\
\hline$>2$ & $858(26 \%)$ & $414(24 \%)$ & $250(27 \%)$ & 194 (31%) \\
\hline \multicolumn{5}{|l|}{ Vital variables } \\
\hline Systolic blood pressure, Median (IQR) & $91(84-96)$ & $96(93-97)$ & $87(84-89)$ & $74(66-79)$ \\
\hline Diastolic blood pressure, Median (IQR) & $55(47-63)$ & $60(53-67)$ & $54(47-60)$ & $44(37-50)$ \\
\hline Heart rate, Median (IQR) & $82(68-100)$ & $81(68-96)$ & $85(68-104)$ & $86(68-104)$ \\
\hline \multicolumn{5}{|l|}{ Shock Index (SI), n (\%) } \\
\hline SI, Median (IQR) & $0.9(0.8-1.2)$ & $0.9(0.7-1.0)$ & $1.0(0.8-1.2)$ & $1.2(0.9-1.5)$ \\
\hline \multicolumn{5}{|l|}{ Time of contact } \\
\hline Day (07:00-14:59) & $1,536(47 \%)$ & 779 (45 \%) & $462(50 \%)$ & 295 (47 \%) \\
\hline Evening (15:00-22:59) & $1,152(35 \%)$ & $640(37 \%)$ & 297 (32 \%) & $215(35 \%)$ \\
\hline Night (23:00-06:59) & $580(18 \%)$ & $306(18 \%)$ & $161(18 \%)$ & $113(18 \%)$ \\
\hline \multicolumn{5}{|l|}{ Mortality (Overall) } \\
\hline 7-day mortality, n (\%) & 489 (15 \%) & $150(9 \%)$ & $153(17 \%)$ & 189 (30 \%) \\
\hline 30-day mortality, n (\%) & $731(22 \%)$ & $264(15 \%)$ & $233(25 \%)$ & $234(38$ \%) \\
\hline 90-day mortality, n (\%) & $922(28 \%)$ & $363(21 \%)$ & $286(31 \%)$ & $273(44 \%)$ \\
\hline
\end{tabular}

${ }^{a}$ Values expressed as total number (fraction) and medians [25 percentile-75 percentile] as appropriat. Chi-squared test for categorical variables and Kruskal-Wallis test for continuous variables. ${ }^{\mathrm{b}} 128$ observations missing

study, increased in the years 2009-2011 compared to the years before. The interpretation of this increase is not obvious and may be confounded by factors such as greater awareness of critical illnesses and per se increased measurement of vital parameters.

We found higher IRs among elderly males, compared to women. Moreover $55 \%$ of our cohort represented patients aged 65 years or more, increasing with decreasing SBP level. Accordingly, a large Canadian study analyzed 34,454 ED visits by older adults (>65 years), accounting for $22 \%$ of the total ED visits in which, $74 \%$ of patient visits were triaged as urgent or emergent [14]. The most common diagnoses (ICD-9 and ICD-10) were nonspecific, relating to "symptoms, signs, and ill-defined conditions" (25\%). Injury and poisoning constituted 17 $\%$ of diagnoses, while diagnoses related to the circulatory system and respiratory system constituted 10 and $9 \%$ of diagnoses, respectively [14].

Comparing these proportions with ICD-10 discharge groups in our study suggest a similar pattern, given the increasing IRs and the dominating ageing proportion of patients. Discharge diagnoses were dominated by injury and poisoning, circulatory system and unspecific diagnoses (symptoms and abnormal clinical/laboratory findings) in our cohort. Other studies, among undifferentiated nontraumatic hypotension in the ED, report sepsis and cardiovascular diseases as common etiologies $[15,16]$. A similar etiological distribution applies for critically ill hypotensive patients in the ICU [1]. This difference could reflect the use of ICD-10 codes, and population-based setting, while others have applied primary clinical assessments and strict inclusion criteria when categorizing 


\section{A Time between systolic blood pressure measurement and ED arrival}

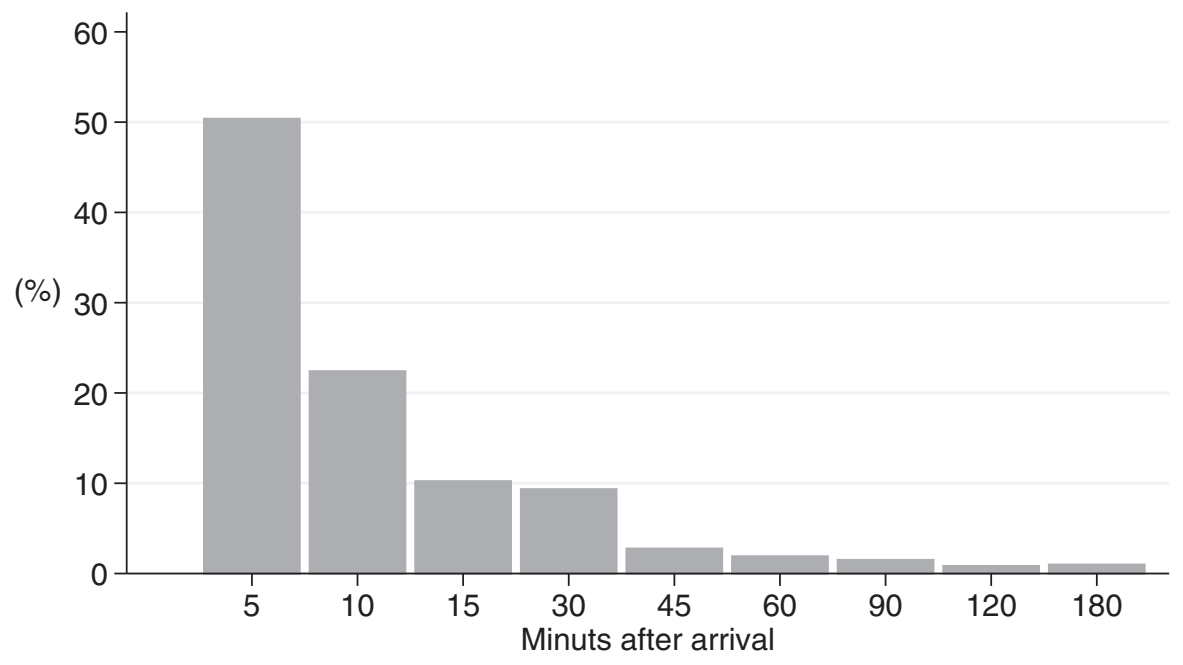

B

Time of arrival to the ED

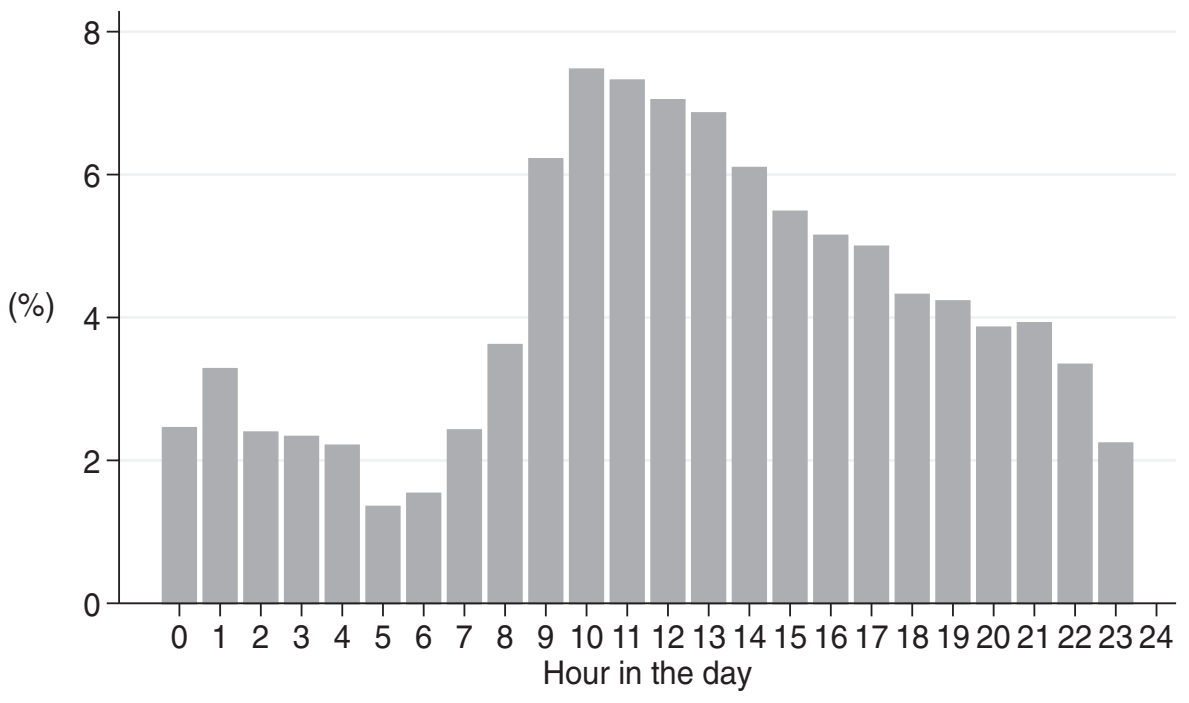

\section{Proportion of cases}

Fig. 2 Histogram illustrating time between blood pressure measurement and arrival to the ED (a) and time of arrival stratified into hours of the day (b)

the etiology. Interestingly, infectious and cardiovascular diseases increased by each decile decrease in SBP level, whereas trauma decreased accordingly. Moreover, we found an increase in discharge diagnoses of infectious and respiratory diseases, disease of the genitourinary system, as well as symptoms, signs, and abnormal clinical and laboratory findings, not elsewhere classified. As a supplementary analysis we applied validated discharge diagnoses for patients with community-acquired infections presenting to the ED (see Appendix: Table 4 for ICD-10 codes validated by Henriksen et al. [17]). Using this algorithm we found a proportion of $13 \%$ with a discharge diagnoses of infection, compared to
$5 \%$ in the initial analysis. The difference reflects our use of merely major ICD-10 groups, as certain infectious diseases (e.g. ICD10-J189 = "pneumonia, unspecified") are grouped under respiratory diseases in the ICD10 system. Applying the validated discharge diagnoses for infections we found a confirmatory increasing trend $(p=0.011)$. Although the data source used is considered a unique information source to carry out epidemiological studies and health service research in our country, the discharge diagnoses among hypotensive ED patients have not undergone validation. The heterogeneous etiological data presented here, should therefore be interpreted bearing this in mind. 


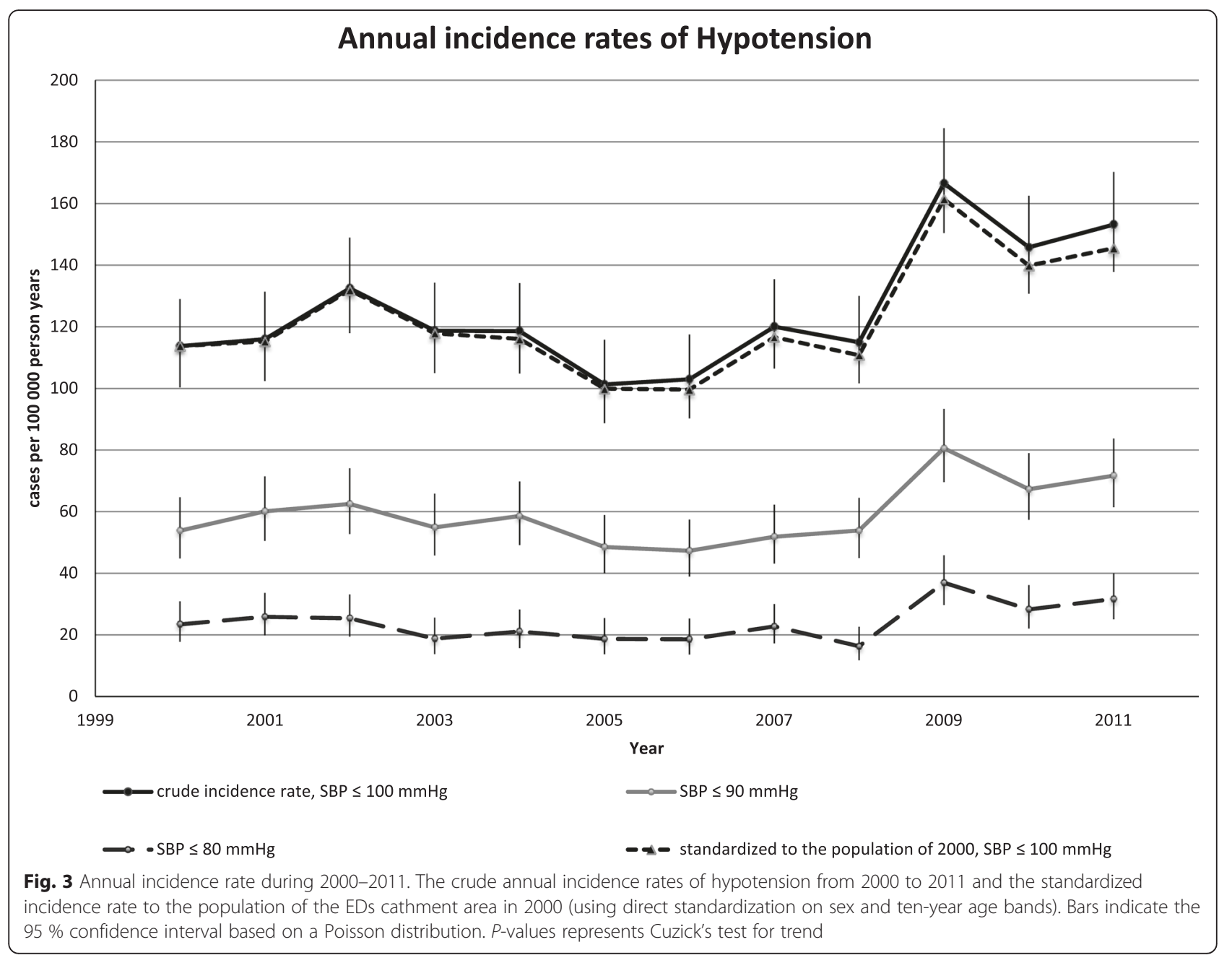

An important finding in this study is the 90-day allcause mortality of $28 \%$. Correspondingly, in-hospital mortality among non-traumatic hypotensive patients, (SBP $\leq 100 \mathrm{~mm} \mathrm{Hg}$ ) is reported to be $10-25 \%[16,18-22]$ while mortality among traumatic hypotensive populations (SBP $\leq 100 \mathrm{~mm} \mathrm{Hg}$ ) are 7-24 \% [23, 24]. As reported by Jones et al. we find an exposure of a single episode of hypotension $(<100 \mathrm{~mm} \mathrm{Hg})$ in the ED setting to portend a possible later adverse outcome [7]. Furthermore, the mortality seem to increase with each decile decrease in SBP as reported previously [19].

We decided to include patient with a first time presentation of SBP $\leq 100 \mathrm{~mm} \mathrm{Hg}$ measured within $3 \mathrm{~h}$ upon arrival. Only 85 patients did not meet this eligibility criterion. Moreover $92 \%$ had their vital values measured within $30 \mathrm{~min}$. All patients in our cohort had a mean SI $\geq 0.9$ suggesting possible acute or critical illness. This could imply, that a great proportion of patients presented with clinical symptoms suggesting critical illness and therefore the ED personal deemed SBP measurement appropriate in order to delineate the hemodynamic stability. Whether a large proportion of our cohort presented with shock (e.g. organ failure or elevated lactate) is of interest, but not feasible based on the available data presented here.

We believe this population-based study provides robust data on the incidence of hypotension in the ED. When hypotension is present, mortality is substantial. Correct diagnosis and resuscitation of patients with hypotension are well-known steps to improve prognosis. Future epidemiological perspectives for research should address the underlying etiology and prognosis of undifferentiated hypotension as this could delineate targeted interventions at ED arrival. At the level of triage, SBP $\leq 100 \mathrm{~mm} \mathrm{Hg}$ should be regarded as a critical finding and the cause of hypotension explored. Future prehospital protocolised management by combining e.g. ultrasound, vital parameters and lactate could further expedite resource allocation and triage of these, often critically ill patients.

\section{Study strengths and limitations}

The Danish public healthcare system, with a complete, independently and prospectively recorded medical 

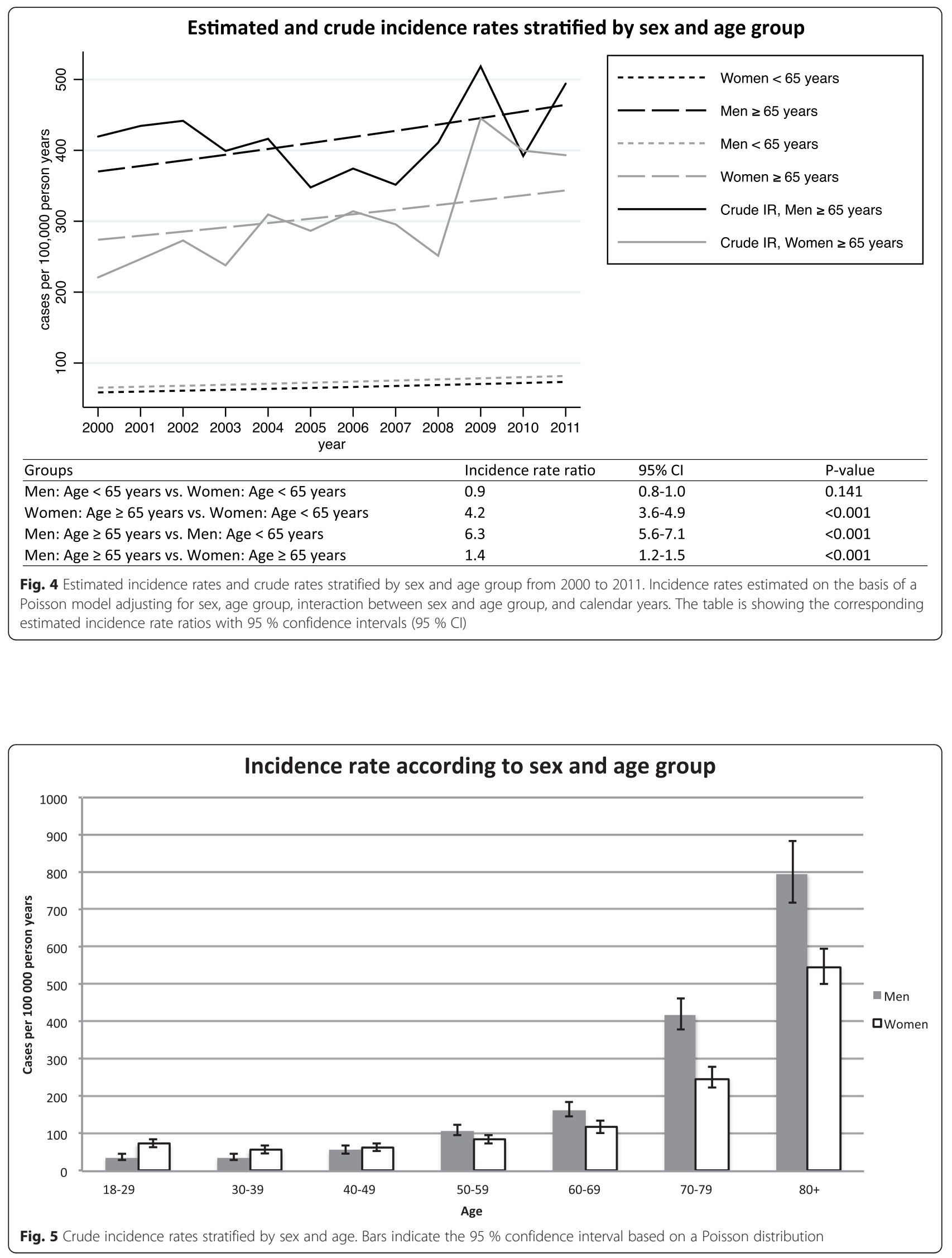
Table 2 Frequency and proportion of primary admission conditions and discarge conditions according to major ICD-10 groups including SBP intervals ${ }^{a}$

\begin{tabular}{|c|c|c|c|c|c|c|c|c|c|c|c|}
\hline \multirow[b]{3}{*}{ ICD-10 groups } & \multirow[b]{3}{*}{ Disease categories } & \multicolumn{4}{|c|}{ Total(\%) SBP $\leq 100 \mathrm{~mm} \mathrm{Hg}$} & \multirow{2}{*}{\multicolumn{2}{|c|}{$\frac{90>\mathrm{SBP} \leq 100 \mathrm{~mm} \mathrm{Hg}}{\text { Discarge }}$}} & \multirow{2}{*}{\multicolumn{2}{|c|}{$80>\mathrm{SBP} \leq 90 \mathrm{~mm} \mathrm{Hg}$}} & \multirow{2}{*}{\multicolumn{2}{|c|}{$\frac{\mathrm{SBP} \leq 80 \mathrm{~mm} \mathrm{Hg}}{\text { Discarge }}$}} \\
\hline & & \multicolumn{2}{|c|}{ Admission } & \multicolumn{2}{|c|}{ Discarge } & & & & & & \\
\hline & & $\mathrm{n}$ & $\%$ & $\mathrm{n}$ & $\%$ & $n$ & $\%$ & $\mathrm{n}$ & $\%$ & $n$ & $\%$ \\
\hline \multirow[t]{3}{*}{ S00-T98 } & $\begin{array}{l}\text { Injury, poisoning, and certain other } \\
\text { consequences of external causes }^{b}\end{array}$ & 805 & 25 & 756 & 23 & 458 & 27 & 192 & 21 & 106 & 17 \\
\hline & Medical (T36-T69, T80-T98) & 186 & 6 & 201 & 6 & 115 & 7 & 59 & 6 & 27 & 4 \\
\hline & Trauma (S00-T35, T70-T79, T90-T99) & 619 & 19 & 555 & 17 & 343 & 20 & 133 & 14 & 79 & 13 \\
\hline 100-199 & Diseases of the circulatory system & 374 & 11 & 489 & 15 & 194 & 11 & 152 & 17 & 143 & 23 \\
\hline R00-R99 & $\begin{array}{l}\text { Symptoms, signs, and abnormal } \\
\text { clinical and laboratory findings, } \\
\text { not elsewhere classified }\end{array}$ & 545 & 17 & 457 & 14 & 251 & 15 & 122 & 13 & 84 & 13 \\
\hline J00-J99 & Diseases of the respiratory system & 229 & 7 & 304 & 9 & 153 & 9 & 95 & 10 & 56 & 9 \\
\hline K00-K93 & Diseases of the digestive system & 209 & 6 & 295 & 9 & 130 & 8 & 98 & 11 & 67 & 11 \\
\hline Z00-Z99 & $\begin{array}{l}\text { Factors influencing health status and } \\
\text { contact with health services }\end{array}$ & 641 & 20 & 245 & 8 & 137 & 8 & 72 & 8 & 36 & 6 \\
\hline A00-B99 & Certain infectious and parasitic diseases & 63 & 2 & 150 & 5 & 69 & 4 & 38 & 4 & 43 & 7 \\
\hline E00-E90 & $\begin{array}{l}\text { Endocrine, nutritional, and metabolic } \\
\text { diseases }\end{array}$ & 156 & 5 & 135 & 4 & 75 & 4 & 39 & 4 & 21 & 3 \\
\hline F00-F99 & Mental and behavioral disorders & 107 & 3 & 129 & 4 & 90 & 5 & 25 & 3 & 14 & 2 \\
\hline No0-N99 & Diseases of the genitourinary system & 33 & 1 & 83 & 3 & 46 & 3 & 24 & 3 & 13 & 2 \\
\hline G00-G99 & Diseases of the nervous system & 42 & 1 & 63 & 2 & 41 & 2 & 16 & 2 & 6 & 1 \\
\hline D50-D89 & $\begin{array}{l}\text { Diseases of the blood and blood-forming } \\
\text { organs and certain disorders involving } \\
\text { the immune mechanism }\end{array}$ & 21 & 1 & 53 & 2 & 28 & 2 & 14 & 2 & 11 & 2 \\
\hline C00-D48 & Neoplasms & 2 & 0 & 43 & 1 & 16 & 1 & 14 & 2 & 13 & 2 \\
\hline M00-M99 & $\begin{array}{l}\text { Diseases of the musculoskeletal system } \\
\text { and connective tissue }\end{array}$ & 28 & 1 & 37 & 1 & 23 & 1 & 8 & 1 & 6 & 1 \\
\hline L00-L99 & Diseases of the skin and subcutaneous tissue & 9 & 0 & 19 & 1 & 8 & 0 & 8 & 1 & 3 & 0 \\
\hline O00-099 & Pregnancy, childbirth, and the puerperium & 2 & 0 & 7 & 0 & 3 & 0 & 3 & 0 & 1 & 0 \\
\hline Q00-Q99 & $\begin{array}{l}\text { Congenital malformations, deformations, } \\
\text { and chromosomal abnormalities }\end{array}$ & 0 & 0 & 1 & 0 & 1 & 0 & 0 & 0 & 0 & 0 \\
\hline Total & & 3,266 & 100 & 3,266 & 100 & 1,723 & 100 & 920 & 100 & 623 & 100 \\
\hline
\end{tabular}

a 2 observations missing, ${ }^{\mathrm{b}} \mathrm{S} 00-\mathrm{T} 98$ ICD-10 codes divided into trauma and medical conditions 
Table 3 Frequency and approximate rounded proportions of discarge conditions according to major ICD-10 groups stratified by year

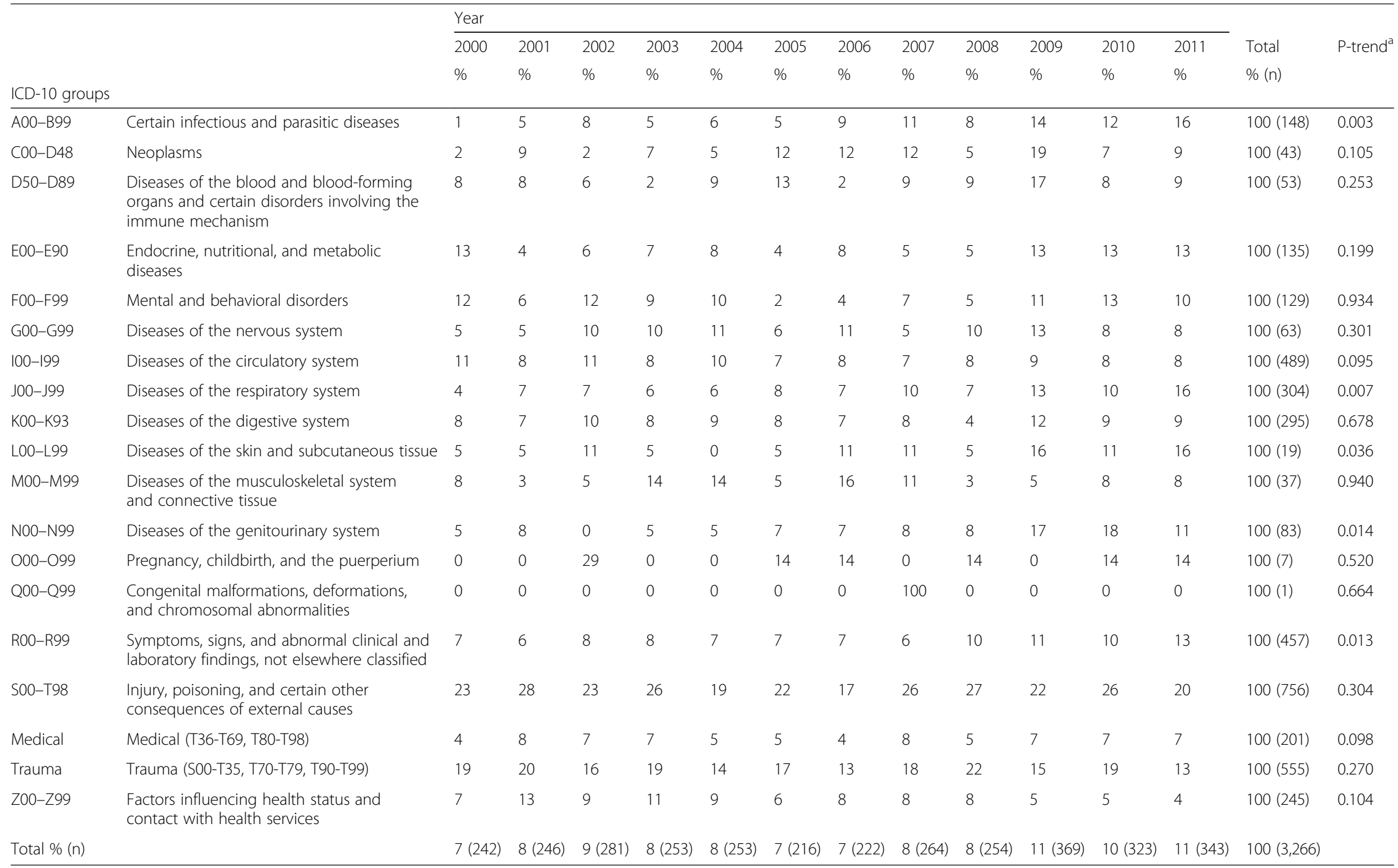




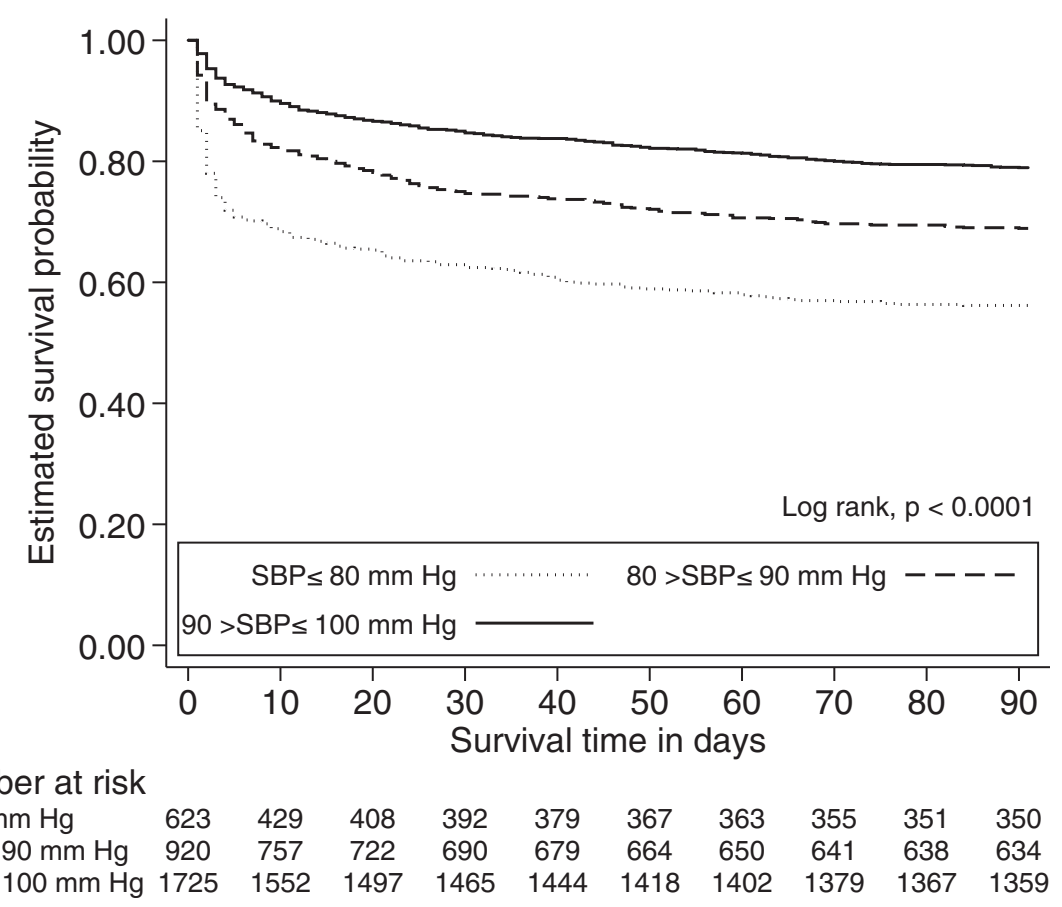

Fig. 6 Kaplan-Meier curves illustrating overall 90-day survival according to different systolic blood pressure levels. Below the curves are listed the number at risk at corresponding intervals in survival time

history, reduced the possible risk of information biases and loss to follow up was not an issue. With the use of the Danish population-based registries we were able to compute quite accurate estimates on the outcomes: incidence, mortality and comorbidity. We chose to use the first contact with hypotension in order minimize bias from repeated measurements. Furthermore we excluded patient with residency outside the catchment area and a previously reported admission with SBP $\leq 100 \mathrm{~mm} \mathrm{Hg}$ in the years 1998-99 in order to avoid possible overestimation of the IRs.

Several issues and limitations should be considered when interpreting our results. Our single center design limits the generalizability of our findings. Although our ED is the only on serving this part of Denmark, the presence of "market share" within the bordering of other ED catchment areas in Denmark is a possibility. We are not able to adjust for possible hypotensive patients living in our catchment area, who have had contact to other hospitals. However, we have excluded patients living in municipalities outside of our ED catchment area and thereby minimized this proportion $(n=516$, Fig. 1$)$. The blood pressure measurements were registered prospectively and as a routine documentation and not necessarily for research purposes. However, a great proportion of cases did not have a SBP measured and registered at arrival $(n=273,794)$. These patients suffered from minor complaints where the nurse did not judge a SBP measurement relevant. Patients with medical complaints and trauma severe enough to warrant a SBP measurement are therefore the population of relevance. This must be kept in mind when interpreting our findings.

We acknowledge the possible limitation in the blood pressure measurement as the accuracy of the automatic oscillometric devices and measurement by auscultation can be inaccurate [25]. However, this is still the method used in most clinical and research based settings when describing blood pressure and we therefore find it generalizable.

We further acknowledge the limitations of the etiological characteristics. Ideally, a classification into shock categories could be clinical useful. However, these data were not available in the current dataset. We had missing values on covariates; ICD-codes (2 cases) and HR (128 cases), but not on SBP. Of notion, is the drop in the IR of 2008 , which was caused by an organizational change in the electronic registration of vital parameters in this year.

Finally, our study and results can be influenced and confounded by unmeasured variables such as use of cardio-therapeutic medications known to inhibit the cardiovascular compensatory response in individuals and potentially mask hypotension and bias our estimates, especially among elderly comorbid patients using these medications. During the observation period a physician-staffed mobile emergency care unit was deployed (October 2007) in the pre-hospital setting. Accordingly, increased awareness and change in 
treatment algorithms in certain critical conditions have been introduced (surviving sepsis campaign and percutaneous coronary intervention of myocardial infarction). Although we consider this proportion minimal, the possibility of patients suffering time-dependent illnesses diagnosed prehospitally (e.g. ruptured aneurism, myocardial infarction) and referred directly to a facility within our hospital (e.g. operational theatre or ICU) and thereby bypassing our ED is a possibility we acknowledge. Although there was no structural change in the primary care service, a change in general practitioners' interest in assessing acute clinical conditions (due to the increasing specialization and fragmentation of primary care services) is another possibility we acknowledge.

\section{Conclusion}

We conclude that a presentation with hypotension is a common critical finding among ED patients with an increasing trend. Adverse outcome are substantial carrying a 90-day all-cause mortality of $28 \%$. Using ICD-10 codes, etiological characteristics are diversified both at ED arrival and at hospital discharge. Prospective risk stratified protocols should evaluate the use and impact prognostics of hypotension in triage algorithms, both prehospitally and in the ED setting.

\section{Appendix}

Table 4 ICD-10 codes identifying infections

\begin{tabular}{|c|c|}
\hline Site of infection & ICD-10 discharge diagnosis \\
\hline Central nervous system & $\begin{array}{l}\text { A39.0, A39.2A, A86.9, A87.0, A87.9, B00.3, B00.4, B02.0, B02.2, B02.2A, B02.2B, B91.9, G00.1, G00.8, G00.9, } \\
\text { G00.9A, G01.9, G04.0, G04.2, G06.0, G06.0 F, G06.2, G07.9 }\end{array}$ \\
\hline Lower respiratory tract & $\begin{array}{l}\text { Pneumonia: A31.0A, A48.1, B37.1, J12.0, J13.9, J14.9, J15,J15.0, J15.1, J15.2, J15.4, J15.5, J15.7, J15.8, J15.9, } \\
\text { J17.0, J17.8C, J18, J18.0, J18.1, J18.8, J18.9, J20.9, J20.9A, J21.9, J22.9, J69.0, J69.8, J69.8AOther: A15.0, A15.1, } \\
\text { A15.2, A15.9, B90.9, J40.9, J44.0, J85.1, J85.2, J86.0, J86.9 }\end{array}$ \\
\hline Urinary tract & A41.9B, N10.9, N12.9, N13.6, N30.0, N30.8, N30.9, N39.0, N39.0B \\
\hline Abdominal & $\begin{array}{l}\text { A00.9, A01.1, A02.0, A03.8, A04.3, A04.5, A04.7, A04.8, A04.9, A05.9, A08.0, A08.1, A08.2, A08.3, A08.4, A08.5, } \\
\text { A09, A09.9, B67.0, K35.0, K35.0A, K35.1, K35.1A, K35.9, K57.2B, K57.3, K57.3A, K57.3B, K57.3 F, K57.9A, K65.0, } \\
\text { K65.0A, K65.0G, K65.0 J, K65.8, K65.81, K65.9, K75.0, K80.3, K80.4, K81.0, K81.9, K83.0 }\end{array}$ \\
\hline Cardiovascular & 130.0, 130.1, 130.8, 130.9, 133.0, 133.9, 138.9, 139.8 \\
\hline Skin, muscles, bones & $\begin{array}{l}\text { A46.9, B00.1 A,B00.1B, B37.2, K61.0, K61.0A, K61.1, K61.2, L02.2, L02.2 T, L02.4, L02.4 F, L02.4 K, L02.9, L02.9A, } \\
\text { L03.1, L03.1E, L03.3, L08.8, L08.9, M00.0, M00.2, M00.2A, M00.8, M00.9, M46.3, M46.4, M46.5, M46.5A, M46.9, } \\
\text { M71.1, M866.1, M86.8, M86.9 }\end{array}$ \\
\hline Viral/systemic & $\begin{array}{l}\text { A51.5, A79.9, B00.1, B05.9, B20.4, B20.6, B20.8, B23.0, B23.2, B24.9, B25.8, B25.9, B27.0, B27.9, B50.9, B52.9, } \\
\text { B54.9, B55.0, B58.9, J09.1, J09.9, J10.0, J10.8, J11, J11.0, J11.1, J11.8 }\end{array}$ \\
\hline Unknown & $\begin{array}{l}\text { A40.1, A40.3, A40.8, A40.9, A41.0, A41.1, A41.1A, A41.2, A41.3, A41.4, A41.5, A41.8, A41.9, A49.9A, B37.7, A32.9, } \\
\text { A41.9A, A42.9, A44.9, A48.2, A49.0, A49.1, A49.3, A49.8, A49.9, A68.9, A70.9, A81.2, B00.8, B02.9, B34.0, B34.9, } \\
\text { B36.9, B37.0, B37.8, B80.9, B89.9, B95.5, B95.6, B95.6A, B96.4, B96.5, B96.8, B99.9, R50, R50.0, R50.8, R50.9, T81.4D, } \\
\text { T84.6, T89.9 }\end{array}$ \\
\hline Other sites of infection & $\begin{array}{l}\text { B00.2A, B02.3G, B37.3A, B37.4, B37.8C, E06.0, E06.1, H65.1, H66.0, H66.9, J00.9B, J01.0, J01.1, J01.2, J01.8, J01.9, } \\
\text { J02.0, J02.9, J02.9B, J03.0, J03.9, J03.9A, J04.0, J05.1, J06.9, J36.9, J39.0C, K04.0A, K05.3A, K10.2C, K11.2C, K12.1, } \\
\text { K62.8 L, N41.2, N45.0B, N45.9, N45.9A, N76.4A, O86.8 }\end{array}$ \\
\hline
\end{tabular}




\section{Abbreviations}

CCl: Charlson comorbidity index; Cl: Confidence interval;

ECG: Electrocardiography; ED: Emergency department; ICD-10: International classification of diseases, tenth revision; IR: Incidence rates; IQR: Interquartile range; MI: Myocardial infarction; HR: Heart rate; PRN-number: Personal registry number; Pyar: Person-years at risk; SBP: Systolic blood pressure; SI: Shock index.

\section{Competing interests}

The authors have no conflicts of interest. JGH and Professor in Emergency Medicine ATL are financially supported by an unrestricted grant provided by the philanthropic foundation TrygFonden to University of Southern Denmark. ATL as well as JGH are employed by the Faculty of Health and Medical sciences, University of Southern Denmark.

None of the authors have financial interests in the project.

\section{Authors' contributions}

JGH conceived the study, analyzed and interpreted the data, and drafted the manuscript together with DPH. ATL conceived and coordinated the study as well as performed critical appraisal of the manuscript. AL, CP, DPH and SM contributed to writing, reviewing, and revising the paper. All authors interpreted the data and critically reviewed drafts of the manuscript. All authors edited and approved the final manuscript.

\section{Acknowledgements}

We would like to thank Niels Ibsgaard Agerbek for professional help with all data extraction as well as MD, PhD Stig lønberg Nielsen and MD, PhD Michala Kehrer for their help on the analysis of data.

\section{Funding}

The work was supported by University of Southern Denmark as well as an unrestricted grant from the Philanthropic private fund TrygFonden given to the University of Southern Denmark.

\section{Author details}

'Department of Emergency Medicine, Odense University Hospital, Odense, Denmark. ${ }^{2}$ Department of Respiratory Medicine, Odense University Hospital, Odense, Denmark. ${ }^{3}$ Department of Anaesthesiology and Intensive Care Medicine, Odense University Hospital, Odense, Denmark. ${ }^{4}$ Department of Infectious Diseases, Odense University Hospital, Odense, Denmark.

\section{Received: 24 October 2015 Accepted: 6 February 2016}

\section{Published online: 02 March 2016}

\section{References}

1. Vincent JL, De Backer D. Circulatory shock. N Engl J Med. 2013;369(18):1726-34. doi:10.1056/NEJMra1208943.

2. Havel C, Arrich J, Losert H, Gamper G, Mullner M, Herkner H. Vasopressors for hypotensive shock. Cochrane Database Syst Rev. 2011;5, CD003709. doi:10.1002/14651858.CD003709.pub3.

3. Gaieski DF, Edwards JM, Kallan MJ, Carr BG. Benchmarking the incidence and mortality of severe sepsis in the United States. Crit Care Med. 2013; 41(5):1167-74. doi:10.1097/CCM.0b013e31827c09f8.

4. Ward MJ, Kripalani S, Zhu Y, Storrow AB, Dittus RS, Harrell Jr FE, et al. Incidence of emergency department visits for ST-elevation myocardial infarction in a recent six-year period in the United States. Am J Cardiol. 2015;115(2):167-70. doi:10.1016/j.amjcard.2014.10.020.

5. Pitts SR, Niska RW, Xu J, Burt CW. National Hospital Ambulatory Medical Care Survey: 2006 emergency department summary. National health statistics reports. 2008(7):1-38

6. Holler JG, Bech CN, Henriksen DP, Mikkelsen S, Pedersen C, Lassen AT. Nontraumatic Hypotension and Shock in the Emergency Department and the Prehospital setting, Prevalence, Etiology, and Mortality: A Systematic Review. PLoS One. 2015;10(3), e0119331. doi:10.1371/journal.pone.0119331.

7. Jones AE, Yiannibas V, Johnson C, Kline JA. Emergency department hypotension predicts sudden unexpected in-hospital mortality: a prospective cohort study. Chest. 2006;130(4):941-6. doi:10.1378/chest.130.4.941.

8. Roberts DC, McKay MP, Shaffer A. Increasing rates of emergency department visits for elderly patients in the United States, 1993 to 2003. Ann Emerg Med. 2008:51(6):769-74. doi:10.1016/j.annemergmed.2007.09.011.
9. Kristensen AK, Holler JG, Mikkelsen S, Hallas J, Lassen A. Systolic blood pressure and short-term mortality in the emergency department and prehospital setting: a hospital-based cohort study. Crit Care. 2015;19(1):158. doi:10.1186/s13054-015-0884-y.

10. Rady MY, Smithline HA, Blake H, Nowak R, Rivers E. A comparison of the shock index and conventional vital signs to identify acute, critical illness in the emergency department. Ann Emerg Med. 1994;24(4):685-90.

11. Schmidt M, Pedersen L, Sorensen HT. The Danish Civil Registration System as a tool in epidemiology. Eur J Epidemiol. 2014;29(8):541-9. doi:10.1007/ s10654-014-9930-3.

12. Schmidt M, Jacobsen JB, Lash TL, Botker HE, Sorensen HT. 25 year trends in first time hospitalisation for acute myocardial infarction, subsequent short and long term mortality, and the prognostic impact of sex and comorbidity: a Danish nationwide cohort study. BMJ. 2012;344, e356. doi:10.1136/bmj.e356.

13. Yeh RW, Sidney S, Chandra M, Sorel M, Selby JV, Go AS. Population trends in the incidence and outcomes of acute myocardial infarction. $N$ Engl J Med. 2010;362(23):2155-65. doi:10.1056/NEJMoa0908610.

14. Latham LP, Ackroyd-Stolarz S. Emergency department utilization by older adults: a descriptive study. Can Geriatr J. 2014;17(4):118-25. doi:10.5770/cgj.17.108.

15. Volpicelli G, Lamorte A, Tullio M, Cardinale L, Giraudo M, Stefanone V, et al. Point-of-care multiorgan ultrasonography for the evaluation of undifferentiated hypotension in the emergency department. Intensive Care Med. 2013;39(7):1290-8. doi:10.1007/s00134-013-2919-7.

16. Jones AE, Tayal VS, Sullivan DM, Kline JA. Randomized, controlled trial of immediate versus delayed goal-directed ultrasound to identify the cause of nontraumatic hypotension in emergency department patients. Crit Care Med. 2004;32(8):1703-8.

17. Henriksen DP, Nielsen SL, Laursen CB, Hallas J, Pedersen C, Lassen AT. How well do discharge diagnoses identify hospitalised patients with communityacquired infections?-a validation study. PLoS One. 2014;9(3), e92891. doi:10.1371/journal.pone.0092891.

18. Jones AE, Stiell IG, Nesbitt LP, Spaite DW, Hasan N, Watts BA, et al. Nontraumatic out-of-hospital hypotension predicts inhospital mortality. Ann Emerg Med. 2004;43(1):106-13. doi:10.1016/50196064403009284.

19. Jones $A E$, Aborn $L S$, Kline JA. Severity of emergency department hypotension predicts adverse hospital outcome. Ga): Shock (Augusta; 2004.

20. Moore CL, Rose GA, Tayal VS, Sullivan DM, Arrowood JA, Kline JA. Determination of left ventricular function by emergency physician echocardiography of hypotensive patients. Acad Emerg Med. 2002;9(3):186-93.

21. Jones AE, Craddock PA, Tayal VS, Kline JA. Diagnostic accuracy of left ventricular function for identifying sepsis among emergency department patients with nontraumatic symptomatic undifferentiated hypotension. Shock. 2005;24(6):513-7.

22. Seymour CW, Cooke CR, Heckbert SR, Copass MK, Yealy DM, Spertus JA, et al. Prehospital systolic blood pressure thresholds: a community-based outcomes study. Acad Emerg Med. 2013;20(6):597-604. doi:10.1111/acem.12142.

23. Eastridge BJ, Salinas J, MCManus JG, Blackburn L, Bugler EM, Cooke WH, et al. Hypotension begins at $110 \mathrm{~mm}$ Hg: redefining "hypotension" with data. J Trauma. 2007;63(2):291-7. doi:10.1097/TA.0b013e31809ed924. discussion 7-9.

24. Bruns B, Gentilello L, Elliott A, Shafi S. Prehospital hypotension redefined. J Trauma. 2008:65(6):1217-21.

25. Jones JS, Ramsey W, Hetrick T. Accuracy of prehospital sphygmomanometers. J Emerg Med. 1987:5(1):23-7.

\section{Submit your next manuscript to BioMed Central and we will help you at every step:}

- We accept pre-submission inquiries

- Our selector tool helps you to find the most relevant journal

- We provide round the clock customer support

- Convenient online submission

- Thorough peer review

- Inclusion in PubMed and all major indexing services

- Maximum visibility for your research

Submit your manuscript at www.biomedcentral.com/submit 